\title{
40 Jahre Strafrechtsreform
}

Monika Frommel

\section{A. Aufbruchstimmung der 1970er Jahre - noch aktivierbar?}

Vor etwa vierzig Jahren gab es zum ersten Mal in der deutschen Geschichte eine knappe, aber handlungsfähige Mehrheit für einen grundlegenden demokratischen Wandel. Getragen war er nicht, wie man vordergründig wegen des Datums meinen könnte, von den 68ern, sie waren damals noch zu jung, sondern von denen, die das Grauen des Kriegsendes gerade noch erlebt hatten. Aber die jugendliche Aufbruchstimmung wirkte atmosphärisch wie ein Verstärker dessen, was zunächst unbemerkt aber nachhaltig während des wirtschaftlichen Aufschwungs in den 1950er Jahren geschehen war. Die nachwachsende Generation bilanzierte neu und fand, dass der ökonomische Aufschwung zumindest teilweise zu teuer erkauft worden sei durch eine politische Amnesie. Übermächtig wurde das Bedürfnis das einzulösen, was im Zuge des wirtschaftlichen Wiederaufbaus auf der Strecke geblieben war: die individuelle Emanzipation und gemeinsame Anstrengungen, um gesellschaftliche Verhältnisse zu schaffen, welche individuelle Freiheit zumindest zulassen. Heute wissen wir, dass diese Veränderungen der Mentalität prägend waren, auch wenn die Akteure gegen große Widerstände anrennen mussten. Wir wissen aber auch, dass die Provokationen der 68er zumindest auch ambivalent wirkten und neben den reformerischen Impulsen höchst destruktive Effekte hatten. Aber sie zwangen zunächst einmal diejenigen, die in den 1970er Jahre die Politik gestalten konnten und wollten, zur Flucht nach vorne, was sich rückblickend als heilsam erweisen sollte.

Die Kinder und Enkel der 68er finden eine andere Gesellschaft und andere ökonomische und politischen Rahmenbedingungen vor. So gesehen kann sich auch die vor vierzig Jahren konzipierte Große Strafrechtsreform in ihren wesentlichen Prinzipien nur halten, wenn diese Veränderungen bedacht, angemessene Konsequenzen erörtert und das überkommene Konzept verfeinert und verändert wird. Aber zunächst fragen wir einmal nach diesem Konzept und seinen sozialen Bedingungen. Danach interessieren die Motive für die späteren Änderungsgesetzgebungen, etwa den Gesetzen zur Bekämpfung des
Terrorismus, der Drogenpolitik der 1980er Jahre (Änderung des BtMG), der 1997 durch einen überfraktionellen Gesetzesentwurf aller Frauen im Bundestag erfolgten Reform der sexuellen Gewaltdelikte, dem kurz danach ein Gesetz zur Bekämpfung der Sexualstraftäter folgte, das bis heute in jeder Legislaturperiode mehrere Änderungen des Rechts zur Sicherungsverwahrung nach sich zog und dem 6. Strafrechtsreformgesetz vor dem Regierungswechsel 1998. Betrachten wir uns schließlich das Feindbild, das die Gegenwart prägt, dann ist dies der gefährliche Sexualstraftäter. Waren die Reformen 1969 - 1975 eher vom Gedanken der positiven Spezialprävention geprägt, gibt es nun ein Nebeneinander beider Strafzwecke, der Besserung und der Sicherung. Damit polarisieren die strafrechtlichen Sanktionen stärker denn je: entweder reagiert man milde und sucht nach Angeboten oder aber man reagiert hart und sichernd. Was hat sich verändert und welchen Rückhalt haben heute noch die Gedanken der Reformer vor vierzig Jahren?

\section{Reformen und Gegenreformen}

Die Strafrechtsreform 1969 - 1975 - eine kriminalpolitische Schönwetterperiode ${ }^{1}$

Rückblickend war die kurze Zeit zwischen 1969 - 1975 eine kriminalpolitische Schönwetterperiode. Der Zeitgeist war innovativ, die Politik nicht von Ängsten, sondern vom Fortschrittsglauben geprägt. Es gelang, das am Ende des 19. Jahrhunderts erdachte Konzept eines auf den unbedingt erforderlichen Gesellschaftsschutz begrenzten Strafrechts zumindest in Ansätzen zu verwirklichen. Die Widerstände waren groß, aber die Reformer setzten sich - zumindest mit einem wesentlichen Teil ihrer Forderungen - durch. In der Gegenwart hätte ein so konsequent auf Haftstrafenvermeidung setzendes Programm keine Chance mehr. Aber fast alle tolerieren den zurückhaltenden Gebrauch von Haftstrafen, wenn die Alternativen glaubwürdig sind. Protest gibt es nur, wenn es gelingt medial plausibel zu machen, dass ein Mord, ein sexueller missbrauch hätte verhindert werden können, wenn staatliche Organe früher und konsequenter gehandelt hätten. Wie sie dies tun, überlässt man ih- nen. Aber sie müssen ein Konzept vorweisen.

Was also waren die ungewöhnlich günstigen Bedingungen für die liberale Reform? Die Antwort ist einfach: ökonomisch stabile Verhältnisse erlaubten es, mehr Demokratie und mehr Freiheit zu wagen. Begriffe wie „Risikogesellschaft“ waren noch nicht erfunden, weil die damaligen Verhältnisse zu solcher Theorie nicht drängten. Sie waren eher verkrustet und vergangenheitsorientiert, denn risikofreudig. Den zukunftsorientierten Wählern erschien es daher lohnend, eine Politik zu unterstützen, die in allen gesellschaftlichen Bereichen mehr individuelle Freiheit, Toleranz und Risikobereitschaft förderte.

Das herkömmliche Strafrecht und insbesondere viele seiner Repräsentanten waren Ende der 1960er Jahre noch auf eine bisweilen unerträgliche Weise geprägt von ihrer unbewältigten Vergangenheit. Die Sprache wirkte formelhaft und es fehlte eine glaubwürdiges kriminalpolitisches Konzept. Die nachwachsenden Generationen waren sich über Parteigrenzen einig, dass das überkommene Strafrecht grundlegend reformiert werden musste. Eines der Ziele lautete: Beschränkung des Strafrechts auf unerlässlichen Rechtsgüterschutz. Damals meinte dies Entkriminalisierung uns Abschaffung der Delikte gegen die Sittlichkeit. Liest man heute in den Materialien der damaligen Reformgesetzgebung nach, was der E $62^{2}$ bei so umstrittenen Tatbeständen wie Prostitution, Kuppelei und Zuhälterei noch für zeitgemäß hielt, versteht man, wie schwer es die Reformer hatten und wie viele Kompromisse auch damals geschlossen werden mussten. Im Jahre 2008 meinte die Mehrheit des Bundesverfassungsgerichts daher, es sei eine Frage der jeweiligen Kriminalpolitik, welche Rechtsgüter geschaffen und geschützt werden, keine der Straftheorie oder der Strafzweckdebatte innerhalb der Strafrechtswissenschaft. Angesichts dieser Entscheidung zur Verfassungsmäßigkeit des Geschwisterinzests wird man diese Debatte wieder neu aufnehmen müssen. Dies soll aber nicht hier geschehen. Es sei an dieser Stelle lediglich vermerkt, dass das Problem vielschichtig und zurzeit ungelöst ist. 
Wie aber war die Praxis? Wie konsequent stellte die Gesetzgebung auf Rechtsgüterschutz um und wie sieht es heute aus? 1969 wurde die Strafbarkeit des Ehebruchs abgeschafft. Es folgte die Streichung des berüchtigten $\mathbb{1 7 5}$, aber nur teilweise: strafbar blieb bis 1993 die Homosexualität erwachsener mit jungen Männern. Der Glaube, man könne sich mit Homosexualität sozial „infizieren“, blieb also lebendig. Auch die einfache Kuppelei wurde gestrichen, aber auch nur bei Erwachsenen. Jede Handlung, die als Förderung der Prostitution gelten könnte, blieb strafbar. Die Polizei kontrollierte mit Hilfe dieser Unzuchtsnorm das Milieu. Erst 2002 entfiel diese stille Befugnisnorm. Es drängt sich somit der Eindruck auf, dass zahlreiche klassische Sittlichkeitsdelikte in jugendgefährdende Delikte umetikettiert wurden, um dem Denken in Rechtsgüter gerecht zu werden ohne die politischen Mehrheiten zu verprellen. Auch die Reform der strikten Strafbarkeit der Abtreibung blieb ein Dauerthema ${ }^{3}$. Sie wurde erst 1995 vollendet, nach über zwanzig Jahren zähen Kampfes. Mittlerweile verlagern sich die Streitigkeiten in geradezu absurde Nebenschauplätze ${ }^{4}$. Das reformierte Strafrecht der 1970er Jahre schützte also trotz des Bekenntnisses zum Rechtsgüterschutz durchaus noch einige höchst strittige Moralvorstellungen. Besonders auffällig ist \182 StGB in der alten und der seit 2008 geltenden neuen Fassung. Bis 1993 war die Verführung eines unbescholtenen Mädchens unter 16 Jahre strafbar ( $\$ 182$ a.F., gültig bis zur deutschen Rechtsvereinheitlichung 1993). 2008 wird diese norm wieder geändert, und zwar im Sinne einer als Schutz vor Ausbeutung getarnten Unzuchtsnorm: Bei Jugendlichen unter 18 Jahren gilt nämlich neuerdings trotz der 2002 erfolgten Legalisierung der Prostitution wieder ein striktes Verbot der Prostitution (strikte Freierbestrafung in dem 2008 verschärften $\$ 182$ StGB, flankiert mit einem Verbot der Jugendpornographie nach dem ebenfalls 2008 neu eingefügten $\ 184$ c StGB). Unverkennbar bewirken hier feministische Moralismen und konservative Strömungen eine neue generalpräventive Moralstabilisierung, dieses mal angeblich im Namen des Fortschritts (Kampf gegen Ausbeutung $)^{5}$. Das geltende Strafrecht wird also wieder durchtränkt von Normen, die einem emphatischen Verständnis von Rechtsgüterschutz widersprechen und der Ideologie folgen, dass es Aufgabe des Strafrechts sei, plakativ eine bestimmte Moral durchzusetzen. Akteure sind nun konservative Feministinnen auf der euro- päischen Ebene. Sie vertrauen darauf, dass die jeweilige nationalen Gesetzgebung diese rechtsgutlosen Delikte „durchwinken“ wird, weil man angeblich an europäische Rahmenbeschlüsse auch dann gebunden sei, wenn die nationale Gesetzgebung bereits sehr viel bessere Normen zum Schutz gegen Ausbeutung hat. Mir scheint das europäische Argument daher eher vorgeschoben zu sein, aber eben erfolgreich vorgeschoben. Es ist ein neuer Politikstil, mit dem man rechnen muss. Im folgenden soll dieses Thema aber nicht vertieft werden, da es sehr komplex ist nicht nebenbei behandelt werden kann.

Erfolgreich war hingegen die Reform des Sanktionenrechts. Das Ziel lautete: Geldstrafen statt kurzen Freiheitsstrafen, Resozialisierung, wenn eine Freiheitsstrafe unerlässlich war, Diversion (Entpönalisierung) und Vorrang ambulanter Maßnahmen vor stationären: Integration statt Ausgrenzung:

\section{- Alternativen zum Strafrecht,}

\section{- Alternativen zum Strafverfahren,}

Diversion, d.h. Einstellung eines Strafverfahren nach der Bezahlung einer Buße oder einer Konfliktschlichtung, eines TäterOpfer-Ausgleichs,

\section{- Alternativen zur Strafe,}

- Kombinationen von eher helfenden mit allenfalls angedrohten repressiven Sanktionen

- Therapie statt bzw. neben einer Strafe

- Freiheitsstrafenvermeidung, etwa durch Strafaussetzung zur Bewährung bzw. offenen Vollzug

- Vermeidung freiheitsentziehender Maßregeln,

- Vorrang der Wiedergutmachung vor einer lediglich vergeltenden Strafe.

Unter dem Stichwort „Entkriminalisierung der Alltags- und Massenkriminalität“ etablierten sich alsbald Routinen der Einstellung eines Strafverfahrens mit oder ohne Auflagen. Sie prägen bis heute die Praxis der Strafverfolgung. Seltener erfolgt dies unter dem Stichwort „Täter-Opfer-Ausgleich“. Aber auch insoweit könnten sich zumindest in der Zukunft Routinen der Schlichtung und Vermittlung festigen, wenn hinter einer Straftat ein Konflikt zu vermuten ist und dieser nur bedingt durch rein strafrechtliche Interventionen thematisiert und gemildert werden kann. Noch ist die Praxis zurückhaltend und eher widersprüchlich. Aber der Gedanke der positiven Spezialprävention hat sich durchgesetzt. Mittlerweile gibt es eine professionell tätige Straffälligenhilfe. Ihre Aufgabe ist es, etwa durch gut ausgestattete soziale Dienste der Justiz (justizintern: Gerichts- und Bewährungshilfe; oder justizextern: freie Träger der Straffälligenhilfe) die Hilfen anbieten, auf die eine spezialpräventiv orientierte Justiz zurückgreifen muss, wenn sie nach Alternativen zur Strafe bzw. zur Freiheitsstrafe sucht.

Aber das milde Strafklima hielt nur an, wenn der Beschuldigte „Deutscher“ war, nicht drogenabhängig, das Delikt „unpolitisch“ und nicht geeignet war, eine massenmedial vermittelte Empörung auszulösen. Nur dann übernahm die Praxis die liberalen Impulse. Da aber die Masse der Delikte diesen Anforderungen entspricht, erwies sich die Reform insgesamt gesehen als erfolgreich. Die Zahlen zur Sanktionspraxis der deutschen Strafgerichte vor und nach der Strafrechtsreform belegen dies. Bei der Masse der leichten und mittleren Kriminalität setzte sich das Prinzip durch, der Geldstrafe vor der Freiheitsstrafe den Vorrang einzuräumen und die Strafaussetzung zur Bewährung großzügig zu handhaben.

Eigentlich hätte die Politik der Freiheitsstrafenvermeidung dem Projekt Resozialisierung nützen müssen. Aber dies gelang nur teilweise. Die freiwerdenden Kapazitäten standen nicht allzu lange zur Verfügung, da die kriminalpolitische Schönwetterperiode endete und neue Belastungsproben die Umsetzung erschwerten: in den 1980er Jahren setzte der „Kampf gegen illegale Drogen “ mit den Mitteln des Strafrechts ein. Weitere untaugliche Versuche illegale Märkte strafrechtlich zu bekämpfen folgten. Die Gefängnisse füllten sich mit Drogenabhängigen und damit veränderten sich die Rahmenbedingungen des Strafvollzugs erheblich. Zwar wussten auch damals alle Experten, dass sowohl der Drogenhandel als auch die Drogenabhängigkeit so nicht zu stoppen sind. Aber die Wirklichkeit der Strafverfolgung wurde spätestens seit den 1980er Jahren vom strafrechtlich unlösbaren Problem der Drogendelinquenz geprägt. Insbesondere der Straf- und Maßregelvollzug hatte nun mit einer zunehmend schwieriger werdenden Klientel zu kämpfen. Das an und für sich sehr belastbare System der Strafverfolgung wurde systematisch überlastet, und zwar mit Problemen, die mit einem spezialpräventiv ausgerichteten Strafrecht überhaupt nicht zu lösen sind.

Sieht man aber von den Folgen dieser - nicht nur für die Abhängigen, sondern auch für eine liberale Rechtskultur - katastrophalen Drogenpolitik der 1980er Jahre einmal ab 
und betrachtet nur den Umgang mit der sog. klassischen Kriminalität, lässt sich feststellen: die Ende der 1960er Jahre einsetzende Strafrechtsreform bewirkte eine enorme Kapazitätssteigerung des Systems der Strafverfolgung. Selbst wenn sie die intendierten humanistischen Ziele nur ansatzweise erreichte, war sie erfolgreich im Sinne einer Steigerung der sozialen Stabilität und war so gesehen eine adäquate Antwort auf den ersten Modernisierungsschub der Nachkriegszeit. Leider wurde versäumt, den gewonnenen Freiraum im Sinne einer positiven Spezialprävention zu nutzen. Stattdessen akzeptierte man die systematische Überlastung der Strafverfolgungsorgane durch immer neue Aufgaben, welche die Politik kurzsichtig dem Subsystem Strafrecht zuschob. Der Trend der Überlastung hält bis heute an.

\section{B. Liberales Strafrecht in der Defensive}

Gegenreformen und Rituale einer permanenten Änderungsgesetzgebung

Von der Antiterrorismusgesetzgebung 1974 über das 6. Strafrechtsreformgesetz 1998 und den Debatten um die Sicherungsverwahrung bis heute

Bereits im Dezember 1974 zeigten sich die ersten Anzeichen einer langfristigen Klimaverschlechterung. Es war dies die erste Phase der Antiterrorismusgesetzgebung. Sie führte in den 1970er Jahren zu einer Politik der permanenten Änderungsgesetzgebung und setzte dem liberalen Modell eines an konkreten Taten orientierten Tatstrafrechts ein Täterstrafrecht entgegen und erprobte mit zunehmender Routine eine Gesetzestechnik, die es erlaubt, bei bestimmten Verdächtigen (den zu vermutenden „Inneren Feinden“) das liberale Strafrecht zwar als Fassade beizubehalten, aber innerlich auszuhöhlen. Heute wissen wir, dass die damaligen Befürchtungen zumindest teilweise realistisch waren $^{6}$. Bedenkt man, welche Traditionen ein reines Täterstrafrecht im Nationalsozialismus hatte und wie zählebig ein moralisierende Denken in Tätertypologien auch noch in den 1950er Jahre war, wundert die Karriere dieses Bekämpfungsstrafrechts nicht. Bei bestimmten - täterspezifisch definierten Katalogtaten (zunächst dem Organisationsdelikt der Mitgliedschaft in einer terroristischen Vereinigung gem. $\$ 129$ a StGB, später auf alle sog. typischen Taten der sog. Organisierten Kriminalität erweitert) - wurden die strengen Formen des Prozeßrechts entformalisiert. Materielles und Strafprozeßrecht veränderten ihren Charakter. Ermittelt wird bei der OK-Bekämpfung nicht eine individuell zurechenbare Tat, sondern die Zugehörigkeit zu einer Szene. Und allmählich wurde eingeführt, was heute unter dem Stichwort „großer Lauschangriff“ diskutiert und besiegelt wird, die Verpolizeilichung des Strafverfahrensrechts und die Vermischung geheimdienstlicher, polizeilicher und strafprozessualer Befugnisse. „Feindstrafrecht“ ${ }^{\text {7 }}$ soll partiell das ohnehin prekäre liberale Tatstrafrecht ersetzen. Auffällig ist die Neigung, Strafrecht auf Halde zu schaffen: Befugnisse, die sich als überflüssig, durch andere ersetzbar erweisen und solche, die noch nicht genutzt werden, die aber in Krisenzeiten verfügbar sind. Außerdem kann ja jederzeit neues Feindstrafrecht (auch diese Debatte kam wieder auf und ist erst in den letzten Jahren beendet worden) geschaffen werden.

Die Antiterrorismusgesetzgebung wäre nicht so erfolgreich gewesen, wenn nicht bereits 1971 mit dem Betäubungsmittelstrafrecht (BtMG) ein „paternalistisch motiviertes Ausnahmerecht" ${ }^{\text {" }}$ entstanden wäre. Die im politischen Strafrecht zuerst erprobten Techniken wurden auf das Recht zur Bekämpfung der Drogenszenen übertragen und ständig verfeinert. Wer versucht, die herkömmlichen dogmatischen Strukturen im Drogenstrafrecht wieder zu finden, erkennt sehr schnell, daß dies ein Ausnahmerecht ist. Im Jurastudium lernen die künftigen JuristInnen daher auch nicht, was sich in diesem Rechtsbereich tut, sondern sie werden ausschließlich zur Bearbeitung klassischer Delikte ausgebildet und merken erst in der Praxis, daß die Grundregeln des klassisch liberalen Strafverfahrensrechts beim Kampf gegen die Drogenszenen nicht gelten. Immerhin, bei den Sanktionen gegen Konsumenten gibt es positive Entwicklungen. Sie gehen auf Teilreformen seit dem Jahre 1982 zurück, in denen versucht wurde, zwischen Konsumenten und den Händlern zu unterscheiden und zumindest für Therapiewillige Alternativen zum Strafrecht zu eröffnen. Diese Normen werden umgesetzt und auch Methadon-Programme sind erfolgreich. AuBerdem wirkt die durch das Cannabis-Urteil des BVerfG ${ }^{9}$ erzwungene partielle Entkriminalisierung von Konsumenten weicher Drogen. Wurde sie zunächst nur in Ansätzen und regional höchst unterschiedlich umgesetzt, gehört sie mittlerweile zur Routine. Interessant ist die Bildung eines europaweiten Interventionsrechtes. In Umsetzung internationaler Vereinbarungen wird neuerdings hart über das Straßenverkehrsrecht zugegriffen, um die offenen Flanken des Strafrechts über repressives Verwaltungsrecht zu schließen.

Mit der Terrorismusbekämpfung und später mit dem Gesetz zur „Bekämpfung“ der sog. Organisierten Kriminalität (OrgKG 1992), ein polizeitaktisches Kürzel, das im wesentlichen Rauschgiftkriminalität meint, wurden Strategien erprobt und gefestigt, deren Ziel die Vermischung von polizeilich präventiven Aktivitäten und Tätigkeiten im Rahmen der Strafverfolgung ist. Dieser Trend hält bis heute an und prägt die internationale Zusammenarbeit auf diesem Gebiet. Verdeckte Ermittlungen und die Beweisführung über verdeckte Ermittler gehören seither zur Routine. Sie dienen einerseits der Beobachtung von Szenen, welche aus polizeitaktischer Sicht, durchleuchtet werden sollten, und sie schaffen Beweise. Selbstverständlich produzieren diese Techniken auch kontaminierte Beweise. Die Strafverteidigung hat es dementsprechend schwer rechtsstaatliche Standards durchzusetzen, zumal am Ende das erkennende Gericht seine Überzeugung lediglich gut begründen muss. Kurz vor dem Regierungswechsel 1998 schuf ein weiteres Gesetz zur Verbesserung der Bekämpfung der OrgK 1998 die gesetzlichen Grundlagen für den großen Lauschangriff, der nun auch in der StPO verankert ist (nachdem die Polizeigesetze der Länder derartiges bereits vorgesehen hatten). Diese wurden allerdings vom BVerfG (BVerfGE 109, 279 ff.) für teilweise verfassungswidrig erklärt und auf schwerste Straftaten beschränkt. 1998 waren diese Gesetzesänderungen zur Ausweitung polizeilicher Ermittlungsarbeit noch umstritten. Mit den Anschlägen des 11.September 2001 veränderten sich die Debatten zur Inneren Sicherheit derartig, dass sich nun keine Regierungspartei mehr traute, die strikte Trennung der Befugnisse von Geheimdiensten, der präventiv tätigen Polizei und der Strafverfolgung zum Programm zu machen. Die deutsche Gesetzgebung schafft seither scheibchenweise eine polizeiliche Befugnis des BKA nach der anderen und verstärkt die Eingriffsbefugnisse einer präventiv sich mit Informationen versorgenden Polizei. 2001 reagierte auch die rot-grüne Koalition prompt mit zwei sog. „Sicherheitspaketen", die die Eingriffsschwellen in die Freiheitsrechte für Ermittlungen weiter absenkten. Ein Überblick über den Inhalt der Änderungen findet sich bei Jan Markus Schulte in der NK 1/2002. Stefan Reinecke schrieb 2003 eine Biografie: Otto Schily - vom RAF-Anwalt zum Innenminister. Seither kann man sich darüber streiten, wie man die Kontinuität der Sicherheitspolitik 
nach 2001 interpretiert. Änderte sich Otto Schily, setzte er bewusst auf die Verstärkung des Bekämpfungsrechts, um die liberale Demokratie funktionstüchtig zu erhalten? Oder wandelte er sich vom Liberalen zum Machtpolitiker. Die Debatte bestimmt auch in diesen Tagen die Bewertung des Entwurfs des BKA-Gesetzes (Gesetz zur Abwehr von Gefahren des internationalen Terrorismus durch das Bundeskriminalamt). Kurz vor der Verabschiedung im Bundestag erging bereits eine einstweilige Anordnung des BVerfG, um zu garantieren, dass der Verhältnismäßigkeitsgrundsatz bei den Eingriffen ins informationelle Selbstbestimmungsrecht der Bürger und Bürgerinnen gewahrt bleibt. Das BKA soll nun zur Abwehr terroristischer Angriffe, also zur Gefahrenabwehr, in Grundrechte eingreifen dürfen und so weit reichende Maßnahmen durchführen dürfen wie etwa Rasterfahndung, Online-Durchsuchung, Überwachung der Telekommunikation, Akustische und optische Überwachung (vgl. dazu die Kritik von Denkowski in der NK 3/2008).

Aber nicht nur die als bedrohlich wahrgenommene organisierte Kriminalität veränderte in den 1990er Jahren den Zeitgeist.
Auch die Furcht vor der angeblich wachsenden Ausländerkriminalität wurde zu einem Schlagwort, ferner begann die Debatte um die Verschärfung des Jugendstrafrechts erneut aufzuflammen. Zusammenfassend kann man sagen, dass in den 1990er Jahren die kriminalpolitischen Feindbilder immer diffuser wurden. Sie zielten nun bereits auf Alltags- und Jugendkriminalität. Stichworte wie „ausländische Diebesbanden“ oder „gewaltbereite Jugendliche“ bürgern sich ein. 1995 versuchte die SPD erstmals, die Innere-Sicherheits-Debatte mit eigenen Inhalten $\mathrm{zu}$ füllen und griff ein medienwirksames Thema auf: Opferschutz. In immer wieder neu bebilderten Fallbeispielen zeigten fiktive Fernsehdokumentationen, daß das aus dem 19. Jahrhundert überkommene Strafrecht bei Eigentumsdelikten höhere Strafen androht als bei Körperverletzungen. Wer eine Camperin beraubt, müsse mit höherer Strafe rechnen als der, der sie vergewaltigt. Führt ein Täter bei einem Raub eine Waffe bei sich, so habe er mit einer Mindeststrafe von fünf Jahren zu rechnen. Eine vergleichbar hohe Mindeststrafe kenne der Vergewaltigungstatbestand nicht und bei der gefährlichen Körperverletzung sei dies sogar die Höchst- strafe. Die SPD forderte daher vor dem Regierungswechsel 1998 eine Strafrahmenharmonisierung (BT-Dr. 12/6164). Während der rot-grünen Regierungsjahre wurde diese Vorgabe über das 6. StRG hinaus (April 1998) konsequent umgesetzt, insb. bei allen sexuellen Gewalt- und Missbrauchsdelikten sind die Mindeststrafen angehoben worden. $\mathrm{Da}$ es zugleich Milderungsmöglichkeiten gibt (flexibles System), haben die Strafgerichte aber über ihren Beurteilungsspielraum bei der Strafzumessung die Möglichkeit, entweder milde zu reagieren oder hart und sichernd. Auch steht angesichts der hohen Mindeststrafen (zwei Jahre bei Vergewaltigung und schwerem sexuellem Missbrauch) jederzeit die Sicherungsverwahrung zur Verfügung. Im Folgenden soll lediglich der Spielraum der Strafverfolgungsorgane einmal graphisch dargestellt werden. Er wird in der Praxis im Allgemeinen liberal genutzt, auch ist die höchstrichterliche Rechtsprechung sehr restriktiv, aber die Tatsache, dass Gerichte hier einen derartig großen Spielraum haben, zeigt, wie dünn der rechtsstaatliche Boden ist, auf dem wir stehen ${ }^{10}$.

\begin{tabular}{|c|c|c|}
\hline $\begin{array}{l}\text { Opferorientierte Strafverfolgung } \\
\text { von Sexualdelikten }\end{array}$ & Justiz (strafrechtlich) & $\begin{array}{l}\text { Konfliktschlichtung } \\
\text { (außerstrafrechtlich) }\end{array}$ \\
\hline Täterorientiert & $\begin{array}{l}\text { a) hohe Mindeststrafrahmen, negative } \\
\text { Spezialprävention, einschließlich der } \\
\text { Sicherung des Täters, } \\
\text { oder } \\
\text { b) eine flexible Nutzung der Strafmilde- } \\
\text { rungsmöglichkeiten } \\
\text { ggf. unterstützt durch eine frühe Einschal- } \\
\text { tung von Sachverständigen im erkennenden } \\
\text { Verfahren nach } \ 246 a \text { StPO, um sinnvolle } \\
\text { Therapie-Angebote im Rahmen der positi- } \\
\text { ven Spezialprävention zu ermöglichen bzw. } \\
\text { das zweispurige Sanktionen-system zu nut- } \\
\text { zen. }\end{array}$ & 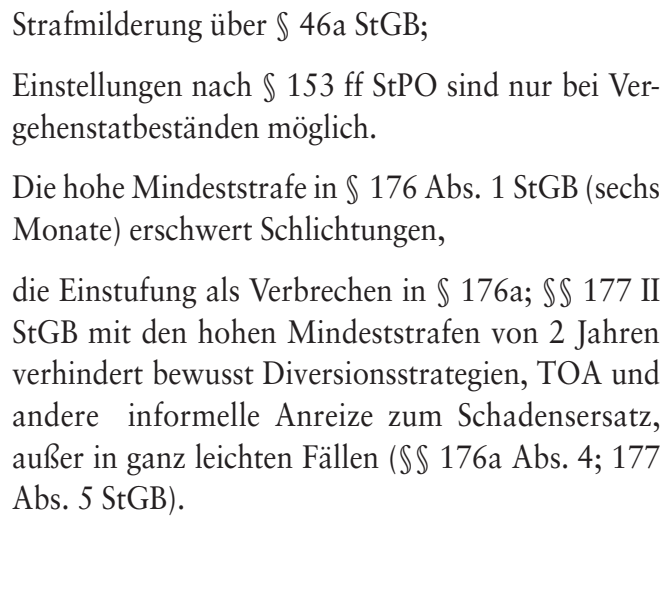 \\
\hline Opferorientiert & $\begin{array}{l}\text { Opferorientierte Strafverfolgung durch } \\
\text { Schwerpunktstaatsanwaltschaften, kom- } \\
\text { munikative Verfahren unter Einbeziehung } \\
\text { der Nebenklage bzw. des Zeugenbeistands, } \\
\text { polizeiliche/ richterliche Videovernehmung, } \\
\text { Zeugenschutz, abgestufte Bewährungsauf- } \\
\text { lagen und frühe Einschaltung von Sachver- } \\
\text { ständigen im erkennenden Verfahren, um } \\
\text { die Qualität der Gefährlichkeitsprognosen } \\
\text { zu verbessern. }\end{array}$ & $\begin{array}{l}\text { a) Schutz von Kindern durch Jugendämter/ Vor- } \\
\text { mundschaftsgerichte; ferner Nutzung des Ge- } \\
\text { waltschutzG, sog. polizeiliche Wegweisung } \\
\text { und } \\
\text { b) Schadensersatz und Schmerzensgeld auch un- } \\
\text { terhalb der Strafbarkeitsschwelle nach } \mathbb{8} 825 \\
\text { BGB }\end{array}$ \\
\hline
\end{tabular}




\section{Warum war das liberale Strafrecht in den 1990er Jahren in der Defensive?}

Probleme der 2. Moderne -Freiheitsgewinne und Sicherheitsverluste in der Risikogesellschaft

Was waren die sozialen Bedingungen für das Gelingen der liberalen Strafrechtsreform und wieso befinden sich ihre Anhänger heute in der Defensive? Die Antwort scheint einfach zu sein: In modernen Industriegesellschaften korrelieren stabile soziale Verhältnisse meist mit stabilen ökonomischen Rahmenbedingungen. Abgesichert wird beides durch eine angemessene sozialstaatliche Absicherung abstiegsbedrohter und hilfsbedürftiger Menschen.

Eine auf Haftstrafenvermeidung setzende und insofern liberale Kriminalpolitik ist also politisch eher durchsetzbar, wenn

1. ein relativ ausgebauter Sozialstaat plausibel den Eindruck vermitteln kann, dass in ausgewählten Bereichen „Hilfe vor Strafe“ gehen soll, jedenfalls in Bereichen, die nicht zur schweren Kriminalität zu rechnen sind, und wenn

2. relativ stabile ökonomische Verhältnisse herrschen.

Die zweite Hälfte der 1960er Jahre stellt so etwas wie eine Epochenschwelle dar. Kennzeichnend ist ein beschleunigter sozialer Wandel, der zunächst unter günstigen ökonomischen Verhältnissen einen Individualisierungsschub auslöste. Gemeint ist damit, dass sich traditionell-gemeinschaftliche Formen des Zusammenlebens zugunsten von Lebensformen auflösen, die weniger von alltäglichen Konformitätszwängen geprägt sind als dies für die herkömmlichen Familienordnungen typisch war. Autonomie, Selbstverwirklichung, veränderte Umgangsformen zwischen den Generationen, mehr Gleichheit, auch zwischen den Geschlechtern, und Multikulturalität sind die positiven Werte dieses Wandels. Negative Begleiterscheinungen sind unübersehbare Desintegrationsprozesse. Sowohl die persönlichen als auch die sozialen Beziehungen zu anderen Menschen sind nicht mehr institutionell gefestigt, gemeinsame Überzeugungen schwinden zugunsten einer Pluralisierung der Lebensstile und einer Relativierung der jeweils einschlägigen Normen. Die Effekte solcher Individualisierungsschübe sind paradox. Sie stellen sehr hohe Anforderung an die Selbststeuerung, garantieren aber nicht, daß entsprechend auch die Mög- lichkeiten verbessert werden, diesen gewachsenen Anforderungen stand zu halten. Die Bildung einer stabilen Identität kann insbesondere dann erschwert sein, wenn sich gleichzeitig die ökonomischen Rahmenbedingungen verschlechtern (Richard Sennett, Der flexible Mensch. Die Kultur des neuen Kapitalismus, dt. Übers. Berlin 1998). Überwogen zunächst - unter günstigen wirtschaftlichen Verhältnissen - die positiven Effekte, schieben sich seit 1989 unübersehbar die negativen Effekte der Risikogesellschaft in den Vordergrund. Sie überlagern die positiven Seiten, die insbesondere Ulrich Beck betont. Wenn der Arbeitsgesellschaft die bezahlte Arbeit ausgeht und sozialstaatliche Kompensationen unbezahlbar werden, zeigen sich die Kehrseiten des zunächst erwünschten Abbaus institutioneller Zwänge. Zwar bleibt sowohl kulturell als auch ökonomisch das Maß der individuellen Gestaltbarkeit hoch. Aber diese neuen Freiheitsspielräume werden nicht mehr vorwiegend positiv erlebt. Denn sie steigern auch das Risiko des Scheiterns. Die selbstgestaltete „Bastelbiographie“ kann in eine „Bruchbiographie“ umschlagen (so Ulrich Beck). Soziale Stabilität wird zur knappen Ressource.

Wendet man ökonomische Stabilitätskriterien kriminalsoziologisch an, dann waren für die etwa bis in die 1960er Jahre datierte 1 . Moderne negative Güter wie Armut, Alkoholismus und Arbeitslosigkeit typisch. Sie wurden neben angeblich biologischen Defekten und sog. Sozialisationsmängeln in der Kriminologie bis in die 1960er Jahre quasi wie kriminogene Faktoren gehandelt. In der seit den 1960er Jahren einsetzenden 2. Moderne haben sich zu diesen negativen Güter neue hinzugesellt: soziale Isolation, Segregation und Subkulturbildung. Sie zeigen sich besonders ausgeprägt in sichtbaren Drogenszenen und bei illegal lebenden, oft ausländischen Drogenhändlern. Und schließlich hat sich mit der ökonomischen Globalisierung des 21. Jahrhundert die soziale Ungleichheit verstärkt. Aber im Gegensatz zu früher treten die ökonomischen und sozialen Probleme mittlerweile so deutlich ins Bewusstsein, dass die Bereitschaft wieder zunimmt, sich mit der zunehmenden sozialen Ungleichheit und den damit einhergehenden Probleme direkt zu befassen, statt über den Umweg einer Inneren-SicherheitsDebatte die Gründe für die Probleme vorwiegend in individuellen Defiziten Einzelner zu suchen. Befragt man im 21. Jahrhundert Bürger und Bürgerinnen, dann sind es nicht mehr Kriminalitätsprobleme, die sie drü- cken, sondern Arbeitsplatzverlust, Altersarmut, Isolation, Umweltzerstörung u.ä. In einer Mediengesellschaft bedeutet dies, dass nicht mehr derjenige die Wahlen gewinnt, der mit der Kriminalitätsfurcht Wahlkampf macht, sondern es setzten sich schlicht andere Themen durch.

Betrachten wir einmal die öffentliche Wahrnehmung der sichtbaren Drogenszene genauer. Sie hat sich in den letzten vierzig Jahren erheblich gewandelt. In den 1970er Jahre wurde Drogenkonsum noch mit Protest und Bewusstseinserweiterung assoziiert. Die Hippies der späten 1960er Jahre lösten zwar die späteren Kriege gegen illegale Drogen und diejenigen, die sie vertreiben und konsumieren, aus, aber sie galten noch nicht wie heute als „Bohème der neuen Unterschicht" ${ }^{11}$, sondern wurden noch als Protestbewegung wahrgenommen. Übertragen wir diesen Aspekt auf den Wandel im Selbstverständnis der Generationen, zeigt sich, dass sich die heutigen „Kinder der Freiheit“ so gesehen erheblich von ihren Müttern und Vätern unterscheiden. Sie protestieren nicht gegen eine saturierte kleinbürgerliche $\mathrm{Ge}$ sellschaft, sondern sie versuchen mehr oder weniger erfolgreich bzw. vergeblich, sich in einer zunehmend flexibilisierenden Welt zu orientieren und zu behaupten. An die Stelle des Protestverhaltens sind Orientierungsverlust und Unsicherheitsgefüble getreten. Aber die Kriminalisierung der Drogenszene hat zugleich abschreckend gewirkt. Heute thematisieren Lehrer nicht mehr illegale Drogen, sondern reden über Alkoholprobleme und klagen über Verwahrlosungserscheinungen. Der beschleunigte soziale Wandel hat nicht nur eine Krise der Städte in der 2. Moderne (Manuel Eisner, Das Ende der zivilisierten Stadt? Campus 1997) nach sich gezogen, sondern eine Veränderung, welche nun auch die Provinz erfasst. Themen wie Verbrechensfurcht und Kriminalprävention (wie immer dieser Topos verstanden wird) sind nicht nur medial erzeugt, sondern auch soziostrukturell bedingt. Gewaltprobleme und eine gestiegene Sensibilität für Gewalt prägen die Diskurse im 21. Jahrhundert.

Die beobachtbaren sozialen Veränderungen prägen die heutigen Anforderungen an ein rationales System der sozialen Kontrolle. Es geht nicht mehr um die Kontrolle von Subkulturen, sondern um Alltagsrisiken. Offenbar verändert sich in einer Gesellschaft, in der zunehmend viele Individuen weniger unter den Zwängen des Familienund Arbeitslebens leiden als vielmehr unter Orientierungsverlust, die Einstellung zum 
Strafrecht. Die gemeinsame Erfahrung sozialer Destabilisierung macht klarere Grenzen erforderlich. Die Einstellungen zu Strafen und zum Strafrecht werden wieder positiver als in der Phase des Protestverhaltens gegen repressive Zwänge einer als überholt angesehenen, vormodernen Gesellschaft. Waren früher punitive Einstellungen Ausdruck einer eher unflexiblen, konservativen Haltung, speist sich der Ruf nach law \& order heute aus ganz verschiedenen Quellen. Zwar gibt es auch in einer hochmodernen Gesellschaft konservative, starre Strafbedürfnisse. Aber sie erklären nicht den gewandelten Zeitgeist. Dieser ist nicht konservativ, sondern er artikuliert eine gewandelte Interessenlage. Eingeklagt werden Sicherheitsinteressen der nicht marginalisierten Mittelschichten. In einer Gesellschaft, in der Negativfaktoren wie Arbeitslosigkeit, Armut oder fehlgeschlagene Sozialisation als „Ursache“ eines kriminellen Verhaltensstils diskutiert werden, ist eine harte, ausgrenzende Kriminalpolitik nicht unwahrscheinlich, aber erst plausibel, wenn sich eine kriminelle Karriere gefestigt hat. Umgekehrt scheinen unangemessen milde Reaktionen auf schwere Norm- und Interessenverletzungen die soziale Notwendigkeit zu verfehlen, den auf Selbstverwirklichung programmierten Individuen Grenzen zu ziehen. Legitimes Strafrecht muß daher besonders genau auf Verhältnismäßigkeit und Fairneß achten. Analysiert man die theoretischen Grundelemente eines liberalen Strafrechts, dann sieht man, dass das überkommene Konzept zwei Elemente verbindet, die nach wie vor aktuell sind: Es berücksichtigt die Notwendigkeit, Grenzen zu ziehen, und mildert dennoch die negativen Folgen vollzogener Freiheitsstrafen durch Strategien der Haftvermeidung. In einer rational geführten Debatte kann sich das Konzept eines liberalen Strafrechts behaupten. Dennoch wird es in der politischen Wirklichkeit auch als Modell von gestern mißachtet.

Die Gründe liegen nur zu einem kleinen Teil auf der Hand. Bekanntlich reagiert Politik kurzfristig auf externen Druck. Empörung und Beschwichtigung sind schneller zu organisieren als effektive Problemlösungen. Diese verlangen nämlich langfristig angelegte Strategien und stabile Verhältnisse, in denen sich Routinen bilden und Kooperationen zwischen verschiedenen Instanzen festigen können. So gesehen scheinen populistische Kriminalpolitiker zu verkennen, dass die Gesellschaft, in der sie leben, so stabil gar nicht ist, wie sie glauben. Denn nur wer die tatsächlich existierenden Probleme bagatellisiert, greift zu Scheinlösungen.

Aber auch die Liberalen in der Defensive müssen sich umstellen. Die Zeiten sind vorbei, in der kriminalpolitische Debatten als Strafzweckdiskussionen geführt werden können. Es geht schon lange nicht mehr um Weltanschauungsfragen, sondern um eine neue praktische und organisatorische Verbesserung des Systems der Hilfen und Sanktionen. Gefordert ist eine Professionalisierung der sozialen Kontrolle. Diese Aufgabe kann nur anhand konkreter Vorschläge angemessen formuliert werden. Strafrecht kann immer nur ein kleiner Ausschnitt in Interventionsprogrammen sein. In dieser Funktion aber hat es durchaus an Bedeutung gewonnen. Wenn dennoch der Eindruck entstanden ist, dass das liberale Konzept versagt habe, dann liegt dies daran, dass das System der Strafverfolgung überfrachtet worden ist mit Aufgaben, für die es nicht geeignet ist (etwa die Kontrolle offener Drogenszenen oder der illegalen Märkte, die mit Stichworten wie „Zwangsprostitution“ und Menschenhandel thematisiert werden). Und es liegt daran, dass das restitutive Element einer opferorientierten Strafverfolgung vernachlässigt worden ist; von den Konservativen, um mit Hilfe der Betroffenen eine Opfer-Empörung gegen Täter zu mobilisieren, und von den Liberalen, da Opferorientierung nicht in ihre Denkgewohnheiten passte. Aber auch Ignoranz hat ihren Preis. In meinen Augen war und ist er zu hoch. Nach 40 Jahren sind wir noch weit davon entfernt, ein professionelles System repressiver, helfender und restitutiver Interventionen anzubieten. Akuter Reformbedarf besteht bei der organisatorischen Vernetzung der helfenden und sanktionierenden Instanzen. Aber trotz zahlreicher Mängel entspricht das heutige System der Strafverfolgung in seiner Grundstruktur den Anforderungen der damaligen Reform. Am schwächsten ausgeprägt ist sowohl theoretisch als auch praktisch - die Idee eines restitutiven Strafrechts ${ }^{12}$. Konsequent durchgeführt würde es sich hierbei um ein Interventionsrecht handeln, das Belange der verletzten Person beachtet und je nach Fallkonstellation entweder auf Strafe zugunsten einer Wiedergutmachung verzichtet oder Strafe und Wiedergutmachung kombiniert. Es ist zu vermuten, dass auch dieser Impuls noch umgesetzt werden wird - trotz der stummen Widerstände der Praxis. Aber gleichzeitig sollte man nicht die auf verschiedenen Ebenen praktizierte Gegenreform ignorieren, welche nicht nur zu einer medialen Kritik an einer zu laschen Strafjus- tiz führt, sondern auch die Alltagspraxis der Justiz erfasst hat und erfassen kann.

Betrachten wir die bis heute wirkenden Effekte. In den 1970er Jahren dominierten pragmatische Fragen vor weltanschaulichen. Die lautlose Reform von unten erreichte justizpraktisch eine nicht zu unterschätzende Effektivitätssteigerung. Die steigenden Zahlen polizeilich registrierter Bagatellkriminalität wurden durch staatsanwaltliche Routinen der Diversion minimiert und konnten „erledigt“ werden, ohne dass die Kapazität der Strafgerichte nennenswert erhöht werden musste. Die Gefängnisse wurden von sog. Gelegenheitstätern entlastet und mit sog. Intensiv- und Wiederholungstätern belastet. Eigentlich hätte dies eine Reform des Strafvollzugs begünstigen müssen, wenn man den Mut gehabt hätte, das Programm der Haftvermeidung konsequent umzusetzen und sich erfolgreich dagegen zu wehren, unlösbare Probleme aufgebürdet zu bekommen, was meint: illegale Märkte mit strafrechtlichen Mitteln (vergebens) zu bekämpfen.

\section{Ausblick}

Vor vierzig Jahren war es ein Generationenwechsel, der die Reformen voran brachte. Auch in Zukunft wird eine neue Generation gestalten. Zwei Projekte erscheinen mir zukunftweisend: das Gewaltschutzgesetz (vgl. hierzu NK 3-2005: Häusliche Gewalt - Wirkungsorientierte Forschung) und das in diesem Heft von Frieder Dünkel vorgestellte Reformgesetz zu „Familiengerichtlichen Maßnahmen bei Gefährdung des Kindeswohls“. Beide Reformprojekte greifen ein strafrechtlich relevantes soziales Problem auf und suchen nach zivilrechtlichen Lösungen. Sie stärken Opferinteressen durch eine vernetzte Intervention. Strafrecht wird nicht ausgeschlossen, aber zunächst einmal verbessert man die Lage der aktuellen und potentiellen Opfer. Die nichtstrafrechtlichen Interventionen zeigen, dass sich Opferorientierung und ein liberales Strafenverständnis nicht ausschließt. Man muss dann aber der alltäglichen Viktimagogie durch konstruktive Angebote entgegen treten. Das liberale Denkmodell der 1970er Jahre wird sich nicht halten lassen, wenn man der Konfrontation mit den Medien, der Gleichstellungspolitik und den opferorientierten Sichtweiden in der Bevölkerung ausweicht und auch im 21. Jahrhundert versucht, Opfer zu neutralisieren, weil ihre Interessen angeblich mit einem liberalen Strafenverständnis nicht kompatibel seien. Dieser Pfad des aus- 
gehenden 19. Jahrhunderts ist nicht mehr vermittelbar. Aber damit sind wieder alle Fragen offen. Hochmoderne Gesellschaften sind nicht punitiv ${ }^{13}$, aber sie sind sehr sensibel für Situationen, in denen sich Menschen besonders vulnerabel fühlen. Wer hier außerstrafrechtliche Lösungen anbietet, kann nur gewinnen und die innovative Grundstimmung der 1970er Jahre ins 21. Jahrhundert retten. Nur wer sich ändert, kann die Traditionen erhalten, die es wert sind. Dies gilt für die Kriminalpolitik und die Gleichstellungspolitik gleichermaßen; denn wir treffen nun auch auf einen publikumswirksamen bevormundenden Feminismus, dem ein glaubwürdiges Gegenmodell entgegen gesetzt werden sollte.

\section{Fußnoten}

1 Diese Passage leitete vor etwa 10 Jahren einmal einen Vortrag für den 22. Strafverteidigertag am 20.03.1998 in Erfurt ein. Damals lag die Strafrechtsreform 30 Jahre zurück und die Prognosen waren eher düster, da die Regierung Kohl lähmend lange jedes reformerische Potential im tagespolitischen Alltag erstickt hatte. Es war damals zu befürchten, dass der irrige Glaube an eine rein strafrechtliche Bewältigung von schweren sozialen Problemen sich halten werde

So auch der Ausblick von Tim Busch, Die deutsche Strafrechtsreform. Ein Rückblick auf sechs Reformen (1969 - 1998), Kieler Diss., 2004. Seit dem Regierungswechsel 1998 scheinen die jeweiligen Gesetzgeber dies selbst zu sehen. Allerdings gibt es Bereiche, in denen sich die Struktur der Sanktionierung erheblich verändert hat, etwa angesichts der Strafschärfungen und der Politik mit der Sicherungsverwahrung bei den so genannten „gefährlichen Sexualstraftätern", was auch damit zu tun hat, dass seit 1997 (Reform der sexuellen Gewaltdelikte) Kriminalpolitik und Gleichstellungspolitik eine neue Verbindung eingegangen sind, welche neue Themen in den Vordergrund spült.

2 Hubert Treiber, Die rückwärtsgewandte Expertenreform - Ausgewählte Ergebnisse einer Cluster-Analyse zum Abstimmungsverhalten in der Grossen Strafrechtskommission, in: KritV 80 (1997), S. 378-399.

Zeithistorisch aufgearbeitet von Ilya Hartmann, Prostitution, Kuppelei, Zuhälterei. Reformdiskussion und Gesetzgebung seit 1870, 2006.

3 Monika Frommel, Lebensschutz, Autonomie und die Grenzen des Rechts, NK 1-2008.
4 Etwa der noch ungeklärten Frage, ob Ärzte, die Schwangerschaftsabbrüche durchführen, Umsatzsteuer bezahlen müssen. Nach den Strafrechtlern bemächtigen sich nun die Finanzämter der Frage und meinen, eine Abtreibung sei mit einer Schönheitsoperation vergleichbar, auch dies ist eine interessante Verschiebung von Weltanschauung ins scheinbar juristisch technische Alltagsgeschäft.

5 In Deutschland wäre ohne die erfolgreiche Polemik von Alice Schwarzer die liberale Position, die Ronald Dworkin, Freedom's Law 1996 klassisch formulierte, konsensfähig geworden. So aber orientierte sich eine populistische Version innerhalb der Frauenbewegung an der Position von Catharine A. MacKinnon, Nur Worte 1994. Für sie müssen Frauen gegen Prostitution und Pornografie kämpfen, da es die Propaganda für die Unterdrückung der Frau als Sexualobjekt sei: eine geradezu absurde Position, welche ignoriert, dass Frauen auch Sexualsubjekte sind. Aber auch in Europa setzten sich die Skandinavierinnen durch, welche eher dieser Variante des sanft kontrollierenden Feminismus zugeneigt sind. Der deutsche Bundestag winkte einen entsprechenden Rahmenbeschluss durch und schuf $2008 \$ 184$ c StGB, eine vermutlich in weiten Teilen verfassungswidrige Norm, jedenfalls soweit sie Darstellungen verbietet. Hingegen kann man es hinnehmen, wenn Jugendliche davor geschützt werden, dass sie in kompromittierender Form für den Rest ihres Lebens im Internet auffindbar sind. Dann muss man die Norm als Darstellerschutz uminterpretieren und dem Gesetzgeber zugestehen, dass er Datenschutz unter falscher Flagge ausgewiesen hat. Wie sich die Praxis entwickeln wird, ist noch völlig offen.

6 Michael Buback, RAF und die Justiz, 2008. Wie konsequent die Strafjustiz sich vom Geheimwissen der anderen Strafverfolgunsorgane manipulieren ließ, zeigt die bittere Bilanz des Sohnes des in Karlsruhe 1977 ermordeten Generalbundesanwalts Siegfried Buback. Es spricht alles dafür, dass wir nie erfahren werden, wer damals tatsächlich geschossen hat. Diejenigen, welche als seine Mörder verurteilt worden sind, konnten dies nur über die mittäterschaftliche Zurechnung (und zwar einer weiten subjektiven Auslegung von $\$ 25$ II StGB - nach dem Tatplan, nicht der Tathandlung). Sie sind also als RAF zur Verantwortung gezogen worden, nicht weil sie geschossen haben. Dies wurde aber auch nicht offen zugegeben. Michael Buback zeigt überzeugend, dass der Geheimdienst mehr wusste und auch die Staatsanwaltschaft Informationen verschleierte, so dass das OLG Stuttgart nur sehr vordergründig informiert worden ist. Vielleicht erklärt dies die ambivalente Botschaft, welche damals Befürworter und Kritiker in heillose Debatten trieb.

7 Ich erspare mir hier die unsägliche Debatte, welche Günter Jacobs mit seiner unklaren Mi- schung aus Deskription und Affirmation ausgelöst hat. Sie ist m.E. auch als Sackgasse erkannt worden. Aber historisch war dieses Denken in Freund-Feind-Zuschreibungen noch präsent. Es war auch präsent, als es darum ging, die RAF auszuschalten und den Sympathisantensumpf "trocken“ zu legen. Glücklicherweise ist es langfristig gelungen die RAF zu entmystifizieren. Der Aufwand war aber enorm.

8 Cornelius Nestler, Grundlagen und Kritik des Betäubungsmittelstrafrechts, in: Arthur Kreuzer (Hrsg.), Handbuch des Betäubungsmittelstrafrechts, München 1998, S. 697-860, (703).

9 BVerfGE 90, 145, BVerfG NJW 1994, 1577.

10 Monika Frommel, Geschlechtsspezifische Aspekte der Kriminalitätsbelastung und Kriminalitätsfurcht aus Opfer- undTäterperspektive; in: „Handbuch der Kriminalprävention“, Loseblatt-Sammlung, Robert Northoff (Hrsg.), 1. Aufl. 1997, 6. Lieferung 2005, Nomos Verlag Baden-Baden. Vgl. ferner dies., Sexualdelikte, in: Robert Northoff (Hrsg.), in: „Handbuch der Kriminalprävention“, Loseblatt-Sammlung 1. Aufl. 1997, 7. Lieferung 2006.

11 so Henner Hess, Die Zukunft des Verbrechens, KJ 1998, S. 145-161.

12 Klaus Sessar, Wiedergutmachen oder strafen. Einstellungen in der Bevölkerung und der Justiz, 1992. Mittlerweile haben sich zwar die Normen geändert, so dass ein restitutives Vorgehen für die Praxis auch bei Erwachsenen leicht wäre und auch den Opfern zugute kommen könnte. Aber die zurückhaltende Nutzung zeigt, dass dieser Gedanke bei Strafjuristen nach wie vor noch nicht verankert ist, obgleich die Bevölkerung eigentlich offen für eine derartige Verarbeitung von Straftaten wäre, vgl. etwa zur Idee einer Verknüpfung von Schadensersatz und Genugtuung mit den Zielen eines Strafverfahrens: Jan Markus Schulte, Die materielle Wiedergutmachung im Strafverfahren, Kiel Diss. 2005. Am 1.01.2008 hat Österreich eine Reform des Strafverfahrensrechts durchgeführt, welche Wiedergutmachung zu einem eigenständigen Strafzweck erklärt. Vgl. hierzu das Heft der Neuen Kriminalpolitik: „Opferorientierte Strafverfolgung in Österreich“, NK 1-2008.

13 Reuband, NK3-2006, S. 99 - 103 hat gezeigt, dass es ein Mythos sei, wenn man glaubt die Punitivität der Bevölkerung könne die ergangenen Sicherheitsgesetze erklären. Die Verbrechensfurcht in Ost- und Westdeutschland sinkt seit 2002. Dies bedeutet nicht, dass die Erwartung an eine professionelle soziale Kontrolle gesunken sei, aber die Bevölkerung erwartet keinen blinden Aktionismus. Wer ihn dennoch betreibt, muss dies selbst verantworten. 